\title{
EFECTOS DE LA INNOVACIÓN EN LOS PRODUCTORES ORGÁNICOS DE AREQUIPA
}

\author{
EFFECTS OF INNOVATION IN THE ORGANIC \\ PRODUCERS OF AREQUIPA
}

\author{
Anatolia Hortencia Hinojosa Pérez \\ Universidad Católica San Pablo, Arequipa, Perú
}

\section{Resumen:}

El objetivo de este estudio fue conocer los efectos de la innovación, transferidos a través de los Centros de Innovación Tecnológica (CITE), a los productores orgánicos de Arequipa. Fueron evaluados 68 productores orgánicos, de los cuales el $62,90 \%$ son mujeres, con un rango de edad entre 24 y 64 años, y una edad promedio de 44 años, el $62,91 \%$ tiene nivel de instrucción de primaria y secundaria, el $41.93 \%$ de las familias tienen entre 4 y 5 miembros en su familia, y el $38,71 \%$ solo tienen a 2 miembros de la familia que trabajan en la unidad productiva. Se utilizaron instrumentos como un cuestionario para conocer los efectos de la innovación, una entrevista no estructurada e información estadística del Gobierno Regional de Arequipa. Los resultados señalan que la innovación de productos, de procesos, así como la innovación organizacional y marketing, tiene efectos positivos en el nivel de productividad (que tiene el fin de incrementar la eficiencia y eficacia en la utilización de los recursos), en el incremento de los ingresos, en la generación de empleo de calidad, mejorando la calidad de vida, no solo de los productores orgánicos, sino también ser ejemplos de la vida real para acrecentar la sostenibilidad económica, social y ambiental. No se ha encontrado relaciones significativas entre el gobierno y los productores orgánicos.

Palabras clave: Innovación, productores orgánicos, Centro de Innovación Tecnológica, políticas de innovación. 


\section{Summary:}

The objective of this study was to know the effects of the innovation, transferred through the Centers of Technological Innovation (CITE), to the organic producers of Arequipa. A total of 68 organic producers were evaluated, of which $62.90 \%$ were women, ranging in age from 24 to 64 years, and a mean age of 44 years, $62.91 \%$ have primary and secondary education levels, $41.93 \%$ of families have between 4 and 5 members in their family, and $38.71 \%$ have only 2 family members who work in the productive unit. We used instruments such as a questionnaire to know the effects of the innovation, an informal interview and statistical information of the Regional Government of Arequipa. The results indicate that product, process, organizational and marketing innovation have positive effects on the level of productivity (which has the purpose of increasing efficiency and effectiveness in the use of resources), in the increase of the income, the generation of quality employment, improving the quality of life, not only of the organic producers, but also to be real-life examples to enhance the economic, social and environmental sustainability. No significant relationships have been found between the government and organic producers.

Key words: Innovation, organic producers, Innovation Technological Center, innovation policy.

\section{Introducción}

A partir del año 2003, la economía peruana ha tenido altas tasas de crecimiento $6.6 \%$ promedio y tuvo una reducción de la pobreza monetaria de $35 \%$. Sin embargo, pese a haber desarrollado un entorno macroeconómico favorable, el Perú convive con indicadores que muestran un bajo nivel de innovación, una productividad muy baja y heterogénea, altos niveles de desigualdad de ingresos y una cesta exportadora con poca diversificación y aglutinada en recursos naturales. A nivel de la empresa, la productividad está concentrada en las firmas grandes -que son relativamente pocas-, mientras que existe un grupo amplio de micro, pequeñas y medianas empresas (MIPYME) poco productivas especialmente en el sector agrícola (Ministerio de la Producción, 2014).
Con respecto a los productores orgánicos en la región Arequipa, diremos brevemente que son parte de un ecosistema, donde la gestión de la producción fomenta y mejora la salud, pero por falta de capacidad de innovación tecnológica (Arriaga, 2014), no aprovechan aún la demanda mundial creciente de productos ecológicos, por falta de productividad y competitividad. En cuanto al Centro de Innovación Tecnológica CEPRORUI El Taller, éste opera desde el año 2003, promoviendo la innovación que viene impulsando la competitividad agroindustrial de la región (García, 2015).

En ese sentido existe un vacío conceptual que se pretende cubrir con este estudio, debido a que no se cuenta con estudios sobre los efectos de la innovación en los productores orgánicos de la Región 
Arequipa. Pretendemos demostrar que la producción ecológica urge de apoyo y, es un freno a la continua migración, y que además, viene demostrando en la vida real cómo incrementar la sostenibilidad económica, social y ambiental de un país (Coordinadora de Organizaciones de Agricultores y Ganaderos [COAG], 2006).

Carazo (2007) explica que la innovación es la aplicación de nuevas ideas, conceptos, productos, servicios, procesos y prácticas con la intención de ser útiles para el incremento de la productividad y competitividad de las MIPYME. Por su parte Gómez y Calvo (2012), Fernández (2005) y el Manual de Oslo OCDE (2005), consideran cuatro tipos de innovación: Innovación de productos, Innovación de procesos, Innovación de la organización e Innovación de marketing. La importancia de la actividad de innovación, radica en que tiene un efecto positivo en el crecimiento económico y, es como el motor del progreso económico. En el Perú, en los últimos tres periodos presupuestales, se han destinado recursos a las acciones a favor de la ciencia, la tecnología y la innovación tecnológica (CONCYTEC, 2013), pues el año 2011 se invirtieron 15'283,400 de soles para este fin, mientras que el 2012, la suma fue de $177^{\prime} 600,854$, y el 2013 de 45'803,669 soles.

Los Centros de Innovación Tecnológica (CITE), según el (Instituto Tecnológico de la Producción, 2015), los define como "el socio tecnológico de las empresas para promover innovaciones que permiten añadir mayor valor agregado y dar cumplimiento de las normas técnicas, las buenas prácticas y otros estándares de calidad e higiene” (p.1). Los servicios tecnológicos brindados por los CITE contribuyen a incrementar la producción, el valor agregado, los ingresos por ventas, así como a consolidar el empleo de calidad.

Según la FAO (2015), los productores orgánicos son los que tienen la "capacidad de producir alimentos mediante la gestión de los recursos locales sin tener que usar insumos externos o sistemas de distribución de alimentos de los que tienen poco control y a los que tienen poco acceso". Es así que, la producción agraria ligada al territorio podrá subsistir y contrarrestar la tendencia de la desaparición de los productores del campo y al despoblamiento del medio rural, siendo necesario para ello, elevar su productividad que, para Medina (2010), es "la forma de utilización de los factores de producción en la generación de bienes y servicios para la sociedad" señala que tiene el propósito de incrementar la eficiencia y eficacia en la utilización de los recursos.

\section{Aspectos metodológicos}

Aplicamos el método de estudio de caso, respecto a la cual Martínez (2006) argumenta que se trata de una metodología cualitativa que "se ha convertido en la base del desarrollo germinal de las teorías que configuran el campo de la empresa" (p. 174). Asimismo, se ha administrado dos instrumentos, un cuestionario a 68 productores orgánicos de Arequipa, siendo la población de estudio conformada por 120 productores orgánicos, de siete distritos rurales al sur este de Arequipa: Chiguata, Quequeña, Pocsi, Polobaya, Yarabamba, Mollebaya, en la zona de Pachacutec, en el distrito de Cerro Colorado (Sotta, s.f). Los sujetos participantes fueron escogidos mediante 
la técnica de muestreo probabilístico estratificado, con edades entre los 25 y 70 años de edad, con propiedad de tierras que van entre los $0.08 \mathrm{Ha}$ a $5 \mathrm{Ha}$. El cuestionario consta de 20 ítems, el mismo que ha sido validado por juicio de expertos y validez de criterio.

Asimismo, se realizó una entrevista personal no estructurada a algunos productores orgánicos, a representantes del gobierno y al Director del CITE CEPRORUI El Taller. También, se ha utilizado el método de observación directa estructurada, revisión de documentos y de datos estadísticos relacionados con el fenómeno estudiado, entre otros. Los datos se recogieron entre los meses de noviembre y diciembre del 2015 y fueron procesados en el programa SPSS versión 16.0.

\section{Resultados}

Presentamos los resultados en base a la encuesta realizada a los productores orgánicos. Se tiene que el $62,90 \%$ de los encuestados son mujeres. En lo referente a la edad, los que tienen entre 34 a 63 años concentran el $69,35 \%$ de la muestra encuestada. En cuanto al nivel de instrucción, el 62,91\% tiene primaria y secundaria. Igualmente los resultados muestran que el $41.93 \%$ de las familias tienen entre 4 y 5 miembros en su familia, y el $38,71 \%$ solo tiene a 2 miembros de la familia que trabajan en la unidad productiva. Respecto al tipo de vivienda, en el 2008 el $56.45 \%$ eran de adobe, mientras que en el 2015 disminuye en $35.48 \%$. Las casas con material noble, el 2008 representan el $37.10 \%$ y el 2015 aumentan al 59.68\%. También se observa que el $87,10 \%$ afirman que ha recibido capacitación de parte del CITE, igualmente el $75,85 \%$ ha recibido asistencia técnica. Se observa también que el 70,97\% se ha beneficiado del proceso de reconversión a la producción orgánica.

El presente estudio, demuestra que la experiencia de organizar la oferta orgánica, ha surtido efectos óptimos, pues según refieren los encuestados, se han instalado "pequeñas parcelas piloto, donde se validaron el cultivo de hortalizas como: lechugas, apios, porros, acelgas, zanahorias, rábanos, nabos...", y se ha implementado nuevas tecnologías, donde "aprendimos a aplicar abonos e insumos orgánicos, el manejo y control de plagas y enfermedades y la siembra en forma escalonada de las hortalizas en zonas alto andinas".

Asimismo, se ha conseguido mejorar la estructura de la organización, con la instalación de dos centros de acopio que les permitió dar valor agregado, mejora de la calidad y la presentación final de sus productos para la venta. Igualmente, la implementación del canal comercial les facilitó su participación quincenal en la feria "Verde Thani". Al respecto, una asociada declara: "Antes mi producto se malograba en el campo y me propuse venderlo yo misma y así aumenté mis ingresos". Igualmente, otra asociada manifiesta: "Para mi Verde Thani es brindar salud y vida al público consumidor, también nosotros estamos sanos porque comemos natural, además Verde Thani es una familia porque cada sábado estamos juntos...”. 


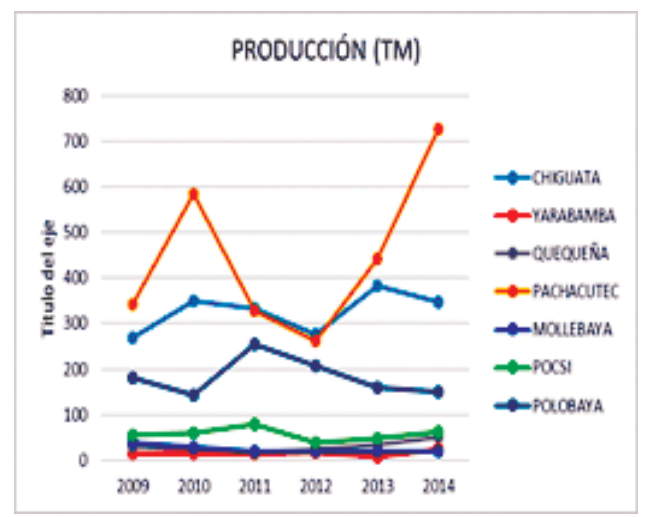

Figura 1.

Producción en TM (GRA, 2015)

Según el Director del CITE CEPRORUI El Taller, señala que a través del Centro de Innovación Tecnológica CITE que dirige se ha apoyado en la innovación de productos, de procesos, organizacional e innovación de marketing, logrando elevar la productividad en un $50 \%$, consiguiendo el aumento del ciclo productivo anual de uno a tres, reduciendo los desperdicios en $15 \%$, mejorando el rendimiento por hectárea y aumentando el ingreso de los pobladores en un $100 \%$.

Por otro lado, el representante de la Municipalidad del distrito de José Luis Bustamante y Rivero, sostiene que apoya estrategias para la apertura de la "Feria Verde Thani”, dando permisos para su funcionamiento en parques y avenidas del distrito. Por su parte el representante del Ministerio de la Producción manifiesta que actualmente vienen aplicando el Plan Nacional de Diversificación Productiva implementando CITE Públicos y que por ahora no cuentan con presupuesto para apoyar a los CITE privados como es el caso de CITE CEPRORUI el Taller.

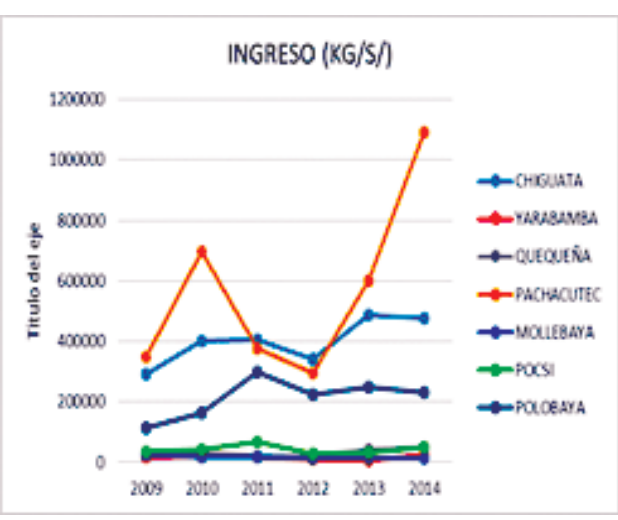

Figura 2.

Ingresos (GRA, 2015)

En base a datos estadísticos de la campaña agrícola, de la Gerencia Regional de Agricultura (2015), se ha demostrado que la producción en toneladas métricas (ver Figura 1) de productos orgánicos como las hortalizas, tiene altibajos durante el periodo 2009 al 2015, pero con una tendencia creciente. También se observa en la Figura 2, que los ingresos en los siete distritos que conforman la unidad de estudio tienen tendencia creciente al igual que la producción.

\section{Conclusiones}

Podemos concluir señalando que los productores orgánicos que se han capacitado, que han recibido asistencia técnica y se han favorecido con la reconversión a la producción orgánica la mayoría son mujeres con una edad promedio de 44 años, y con un nivel de instrucción de primaria y secundaria. Podemos observar también que uno de los efectos de la innovación es la mejora de su tipo de vivienda, por consiguiente una mejora en el nivel de vida. 
Asimismo, se observa que los productores orgánicos, gracias a la innovación de productos, procesos, la innovación organizacional y la innovación de marketing, transferida por el CITE CEPRORUI el Taller, han innovado en la organización de la oferta orgánica, en la planificación de la producción, en la implementación de nuevas tecnologías, en la instalación de dos centros de acopio y en la implementación de canales comerciales con una estrategia de promoción y comercialización de productos orgánicos; con efectos positivos en el nivel de productividad, en el incremento de los ingresos y en la generación de empleo de calidad para mejorar los niveles de vida de los productores orgánicos.

\section{Referencias}

Arriaga E. (2014). "Estudio de los hábitos de consumo de productos ecológicos de los habitantes de Tierra Estrella”. España: Universidad Pública de Navarra.

Carazo, I. (2007). "Centros de Innovación Tecnológica, un desafío para la microempresa”. Perú: MITINCI.

CONCYTEC (2013). "CONCYTEC: Nuevos tiempos para la CTI, Memoria anual 2012-2013. Lima”. (Documento en formato html). Recuperado el 23 de febrero del 2016 de: http://repositorio.concytec.gob.pe/bitstream/ CONCYTEC/22/1/memoria-institucional-CONCYTEC-2012-2013.pdf

Coordinadora de Organizaciones de Agricultores y Ganaderos (COAG) (2006).
"De la producción convencional a la ecológica”. España, Madrid: COAG.

García A. (2015). "Revisión de documentos y encuesta”. (Documento en formato html). Recuperado el 17 de mayo del 2016 de: https://mail.google.com/ mail/u/1/\#search/agarcia\%4oeltaller. org.pe/14e939c187422c55

Gerencia Regional de Agricultura. (2015). “Campaña Agrícola Regional”. Arequipa: Gobierno Regional.

Gómez, A. y Calvo, J. (2012). “Innovación: Factor Clave del Éxito Empresarial”. Bogotá: Ecoe Ediciones.

Fernández, E. (2005). "Estrategia de Innovación”. España: Thomson.

Instituto Tecnológico de la Producción ITP (2015). "Los Centros de Innovación Tecnológico (CITE)” (Documento en formato html) Recuperado el 15 diciembre 2015 de: http://www.itp. gob.pe/index.php/informacion-de-cite

Manual de Oslo (2005). "Guía para la recogida e interpretación de datos sobre innovación”. Tercera Edición. Comunidad Europea: OCDE y Eurostat.

Martínez, P. (2006). El Método de estudio de Caso, Estrategia Metodológica de la Investigación Científica. "Pensamiento y Gestión”, 20, 165-193.

Medina, J. (2007). "Modelo Integral de productividad una visión estratégica”. Bogotá: Universidad Sergio Arboleda. 
Ministerio de la Producción (2014). "Plan Nacional de Diversificación Productiva. Nuevos Motores para el Desarrollo del País". Lima: Produce.

Organización de las Naciones Unidas para la Alimentación y la Agricultura (FAO) (2015). "Por un Mundo sin hambre, Organic Agriculture”.
(Documento en formato html). Recuperado el o8 de enero de 2016: http://www.fao.org/organicag/oa-faq/ oa-faq7/es/

Sotta, N. (s.f). “Comercialización de Hortalizas Orgánicas en Arequipa, una experiencia de pequeños productores alto andinos". Arequipa: El Taller.

Recibido: 30-12-2016 Aceptado: 23-04-2017 